\title{
Controlling traffic jams by time modulating the safety distance
}

Gaididei, Yu B.; Gorria, C.; Berkemer, R.; Kawamoto, A.; Shiga, T.; Christiansen, Peter Leth; Sørensen, Mads Peter; Starke, Jens

Published in:

Physical Review E

Link to article, DOI:

10.1103/PhysRevE.88.042803

Publication date:

2013

Document Version

Publisher's PDF, also known as Version of record

Link back to DTU Orbit

Citation (APA):

Gaididei, Y. B., Gorria, C., Berkemer, R., Kawamoto, A., Shiga, T., Christiansen, P. L., Sørensen, M. P., \& Starke, J. (2013). Controlling traffic jams by time modulating the safety distance. Physical Review E, 88̈(4), [042803]. https://doi.org/10.1103/PhysRevE.88.042803

\section{General rights}

Copyright and moral rights for the publications made accessible in the public portal are retained by the authors and/or other copyright owners and it is a condition of accessing publications that users recognise and abide by the legal requirements associated with these rights.

- Users may download and print one copy of any publication from the public portal for the purpose of private study or research.

- You may not further distribute the material or use it for any profit-making activity or commercial gain

- You may freely distribute the URL identifying the publication in the public portal 


\title{
Controlling traffic jams by time modulating the safety distance
}

\author{
Yu. B. Gaididei \\ Bogolyubov Institute for Theoretical Physics, Metrologichna str. 14 B, 03680, Kiev, Ukraine \\ C. Gorria \\ Department of Applied Mathematics and Statistics, University of the Basque Country, E-48080 Bilbao, Spain \\ R. Berkemer \\ AKAD University of Applied Sciences, D-70469 Stuttgart, Germany
}

A. Kawamoto and T. Shiga

Toyota Central R\&D Labs, Nagakute, Aichi, Japan

P. L. Christiansen

Department of Applied Mathematics and Computer Science \& Department of Physics, Technical University of Denmark, DK-2800 Kongens Lyngby, Denmark

M. P. Sørensen and J. Starke

Department of Applied Mathematics and Computer Science, Technical University of Denmark, DK-2800 Kongens Lyngby, Denmark

(Received 27 September 2012; revised manuscript received 25 June 2013; published 9 October 2013)

\begin{abstract}
The possibility of controlling traffic dynamics by applying high-frequency time modulation of traffic flow parameters is studied. It is shown that the region of the car density where the uniform (free) flow is unstable changes in the presence of time modulation compared with the unmodulated case. This region shrinks when the speed-up of cars does not exceed some critical value and expands in the opposite case. The flux of the time-modulated flow is an increasing function of the amplitude of the modulation for traffic flows whose density is larger than $1 / h$ where $h$ is the safety distance in the nonmodulated case, while it is a decreasing function in the opposite case. In other words, the safety distance time modulation facilitates car propagation in the case when the mean distance between cars in the congestive traffic is less than $h$ and hinders it when the neighboring cars in the flow are well separated. A link between a microscopic description and the macroscopic fundamental diagram is established.
\end{abstract}

DOI: 10.1103/PhysRevE.88.042803

\section{INTRODUCTION}

Traffic flow control is one of the major issues of our civilization. Traffic jams are common phenomena on roads. The mathematical modeling of traffic flow dynamics has a long history (see, e.g., reviews [1-8]). It is now widely accepted (see, e.g., [7,8]) that traffic flow can be considered as a particular example of collective nonequilibrium behavior of asymmetrically coupled elements and that many collective phenomena such as nonequilibrium phase transitions, nonlinear dynamical behavior, bifurcations, and pattern formations are inherent features of traffic flow models [9,10]. Other examples of asymmetrically coupled elements are intersegmental coordination of the neural networks responsible for generation of bipedal locomotion [11,12], electronic circuits [13], multiple robotic systems [14], etc.

There are two fundamentally different directions in microscopic modeling of traffic dynamics: discrete and continuous ones. Discrete models are formulated in terms of so-called traffic cellular automata (see, e.g., a review paper [7]) when the real traffic is considered in a coarse-grain approximation and discrete in time. One of the first who used this approach were Nagel and Schreckenberg [15] who introduced a stochastic discrete automaton model and by using Monte Carlo simulations showed a spontaneous emergence of traffic jams. As a rule, analytical results can be obtained only
PACS number(s): 05.65.+b, 04.25.-g, 89.40.Bb, 89.75.Kd

for special cases, however, computer simulations performed in the framework of this approach are fast and efficient. The continuous approach describes the microscopic traffic dynamics in terms of time-continuous differential-difference equations. This kind of approach was used in Ref. [16] where an intelligent driver model with empirical boundary conditions was successfully used to analyze data from several German freeways. An interplay between the reaction time of the drivers and the time which is needed to accelerate to a new desired velocity (so-called adaption time) in the traffic dynamics and how it influences the stability of traffic flow is studied in Ref. [17] by means of numerical simulations with a time-continuous car-following model. In our paper we will use one of the continuous car-following models, namely the so-called optimal velocity (OV) model introduced in Ref. [18]. In the framework of this model the vehicles numbered with index $n$ are ordered by their time-dependent position as $x_{n}(t)$ such that $x_{n}(t)<x_{n+1}(t)$. Each driver controls the acceleration by reducing the difference between the car velocity $\frac{d x_{n}}{d t}$ and an optimal velocity $V\left[\mathcal{V}\left(\frac{x_{n+1}(t)-x_{n}(t)-H}{\ell_{0}}\right)+v\right]$ where $V$ and $v$ are constant parameters. The optimal velocity depends on the headway $x_{n+1}(t)-x_{n}(t)$, i.e., the distance from the vehicle in front. Here $H$ is the safety distance between a car and the car that is in front of it. Thus, the safety distance is defined as the distance between cars for which the optimal velocity is 
equal to zero: $\mathcal{V}(0)=0$. The optimal velocity $\mathcal{V}(u)$ is assumed to be expressed as a dimensionless sigmoidal function of the distance between cars $[\mathcal{V}(\infty)=1] . V$ is a speed-up or, in other words, it is a maximal velocity gain, which is achieved for infinitely separated cars, compared to some common velocity $v V$ of the cars with which they move when all the headways are equal to the safety distance. Thus, the quantity $V_{s}=V(1+v)$ gives a speed limit. The characteristic length scale $\ell_{0}$ determines the pace with which the driver reaches its maximal velocity $V_{s}$. Then the differential equation of the model reads

$$
\begin{gathered}
T \frac{d^{2} x_{n}}{d t^{2}}+\frac{d x_{n}}{d t}=V\left[\mathcal{V}\left(\frac{x_{n+1}(t)-x_{n}(t)-H}{\ell_{0}}\right)+v\right], \\
n=1, \ldots, N
\end{gathered}
$$

where $T$ is the reaction time. An interesting artificial system, which has many properties similar to the OV traffic model, was proposed quite recently in Ref. [19] where an inanimate system composed of camphor boats in an annular water channel was studied and several modes of collective motion were observed. The boats move on the water and interact with each other through the concentration of the camphor molecules on the water surface. In this sense the camphor boat system gives an experimental realization of a general chemotactic model of oscillators considered in Ref. [20]. Another interesting system where the ideas of OV model may be used was presented in a recent paper [21]. In this paper experimental results for pedestrians walking in line were obtained, a relation between the instantaneous velocity and spatial headway was established, and the fundamental diagram was constructed.

There is a growing interest in finding efficient ways to control traffic flow dynamics in the development of integrated vehicle management systems using advanced sensing technologies such as millimeter wave radar devices and stereo cameras. With the progress of these technologies vehicles may assist drivers in more aspects of driving operations by compensating for the lack of safety and the loss of efficiency. Quite recently studies appeared on traffic flows with vehicles that possess a control system trying to keep a desired headway: vehicles with adaptive cruise control (ACC) [22-26]. Several microscopic models have been proposed to model the ACC vehicles [22,27,28]. It was shown in Ref. [27] that traffic jams are suppressed for the ACC vehicles that are modeled by linear dynamical equations with a delayed-feedback control. Simulations of merging flows near an on ramp and for random sequences of ACC vehicles and manually steered vehicles were carried out in Ref. [28]. Those studies have been so far conducted based on computer simulations. However, in the automotive market, some ACC technologies have been already introduced for precrash safety. With a current ACC system using millimeter wave radar, one can actually maintain a preset following distance within preselected speeds [29].

The goal of this paper is to show that by applying time modulation of traffic flow control parameters one can effectively reduce the tendency to jam formation. To this end we study the optimal velocity equation (5) where each car $n$ is under the influence of a time-modulated input signal $\eta(t)$, modifying the safety distance $h$. The modification of the safety distance $h$ can be done, for instance, by modulating the preset following distance in the above mentioned ACC system. In this case, the traffic flow dynamics is governed by a generalized optimal velocity equation of the form

$$
\begin{gathered}
T \frac{d^{2} x_{n}}{d t^{2}}+\frac{d x_{n}}{d t}=V\left[\mathcal{V}\left(\frac{x_{n+1}(t)-x_{n}(t)-H-F(t)}{\ell_{0}}\right)+v\right], \\
n=1, \ldots, N .
\end{gathered}
$$

It is assumed that $F(t)$ is a time periodic function

$$
F(t)=F \cos (\omega t),
$$

where $F$ and $\omega$ are the amplitude and the frequency of the safety distance modulation, respectively. By introducing the dimensionless variables

$$
\begin{aligned}
& s_{n}=\frac{x_{n}}{\ell_{0}}, \quad h=\frac{H}{\ell_{0}}, \quad \bar{t}=\frac{V t}{\ell_{0}}, \quad \tau=\frac{V T}{\ell_{0}}, \\
& f=\frac{F}{\ell_{0}}, \quad \Omega=\frac{\omega \ell_{0}}{V},
\end{aligned}
$$

Eq. (1) is written in the form

$$
\begin{gathered}
\tau \ddot{s}_{n}+\dot{s}_{n}=\mathcal{V}\left(s_{n+1}(\bar{t})-s_{n}(\bar{t})-h-\eta(\bar{t})\right)+v, \\
n=1, \ldots, N,
\end{gathered}
$$

where

$$
\eta(\bar{t})=f \cos (\Omega \bar{t}),
$$

and the overdot stands for the time derivative $d / d \bar{t}$. In what follows we will write $t$ instead of $\bar{t}$.

The motivation for the periodically modulated safety distance is based on an analogy to well known physical systems, as, e.g., in the inverted driven pendulum (Kapitza pendulum), modulational instability in optics, and changes of stability regions in mechanical systems due to dynamic loads (airplanes). Important here is that the modulation changes the qualitative behavior of the system. One of the reasons why we decided to investigate the effects of time-periodically input signals on the traffic dynamics in the framework of the optimal velocity model is the possibility to study this problem not only by means of numerical simulations but also to obtain some analytical insight into the problem. We believe that the mathematical methods and techniques used in the present paper can be transferred to obtain insight also into other periodically driven physical systems with a discrete structure and are therefore of interest not only to the traffic community but also to a broader readership.

\section{UNIFORM FLOW}

An ideal and desired traffic flow is one with all cars moving with the same velocity $v$ and keeping the same distance $\ell$ between nearest neighboring cars. Such a flow is said to be uniform or free. In the case when the cars are moving along the closed curve of the length $L$ such that the periodic boundary conditions

$$
s_{n+N}=s_{n}+L
$$

are imposed, the uniform traffic flow solution (free flow), which is a steady-state solution of Eqs. (5) reads

$$
S_{n}(t)=n \ell+X(t) \quad \text { with } \quad \ell=\frac{L}{N} .
$$


Here the function $X(t)$ satisfies the equation

$$
\tau \ddot{X}+\dot{X}=\mathcal{V}(\delta-\eta(t))+v,
$$

where the parameter $\delta=\ell-h$ denotes the mismatch between the safety distance $h$ and the mean distance between neighboring cars $\ell$. The question is under which conditions is the uniform traffic flow stable or not, anticipating that an unstable uniform flow will lead to traffic jams. In the nonmodulated case where $f=0$, a standard linear stability analysis shows that for $\mathcal{V}^{\prime}(\delta)>0$ the free flow is stable when the reaction time is shorter than the critical reaction time $\tau_{c}$,

$$
\tau<\tau_{c} \equiv \frac{1}{2 \mathcal{V}^{\prime}(\delta)}
$$

For the opposite inequality the free flow becomes unstable $[18,30]$. The inequality $\mathcal{V}^{\prime}(\delta)<0$ corresponds to an inadequate behavior of the driver: the driver accelerates when the headway decreases.

Let us consider now the case with a safety distance modulation, i.e., $f \neq 0$. In order to investigate the stability of the uniform flow solution we assume that $s_{n}(t)=S_{n}(t)+\psi_{n}(t)$ and linearize Eq. (5) with respect to $\psi_{n}(t)$. As a result we obtain

$$
\tau \ddot{\psi}_{n}(t)+\dot{\psi}_{n}(t)=\mathcal{V}^{\prime}(\delta-\eta(t))\left[\psi_{n+1}(t)-\psi_{n}(t)\right] .
$$

The stability is analyzed by considering solutions of the linear system (11) of the form

$$
\psi_{n}(t)=\Psi_{j}(t) \exp \left\{i \frac{2 \pi j}{N} n\right\}, \quad j=0, \ldots, N-1 .
$$

Insertion of Eq. (12) into Eq. (11) leads to

$$
\tau \ddot{\Psi}_{j}(t)+\dot{\Psi}_{j}(t)=\mathcal{V}^{\prime}(\delta-\eta(t)) \gamma_{j} \Psi_{j}(t),
$$

where the notation

$$
\gamma_{j}=\exp \left\{i \frac{2 \pi j}{N}\right\}-1
$$

is used. Due to Eq. (6), the function $\mathcal{V}(\delta-\eta(t))$ is a timeperiodic function with period $T=2 \pi / \Omega$. By splitting the function $\Psi_{j}(t)$ into its real and imaginary parts

$$
\Psi_{j}(t)=f_{j}(t)+i g_{j}(t),
$$

one can represent Eq. (13) as a set of two coupled Hill equations

$$
\begin{aligned}
\tau \ddot{f}_{j}(t)+\dot{f}_{j}(t) & =\mathcal{V}^{\prime}(\delta-\eta(t))\left(\gamma_{j}^{\prime} f_{j}(t)+\gamma_{j}^{\prime \prime} g_{j}(t)\right), \\
\tau \ddot{g}_{j}(t)+\dot{g}_{j}(t) & =\mathcal{V}^{\prime}(\delta-\eta(t))\left(\gamma_{j}^{\prime} g_{j}(t)-\gamma_{j}^{\prime \prime} f_{j}(t)\right),
\end{aligned}
$$

where $\gamma_{j}^{\prime}=-2 \sin ^{2}\left(\frac{\pi j}{N}\right), \gamma_{j}^{\prime \prime}=\sin \left(\frac{2 \pi j}{N}\right)$ are the real and imaginary parts of the function $\gamma_{j}$, respectively. The stability analysis for solutions to differential equations with periodic coefficients shows (see, e.g., Ref. [31]) that parametric resonance in the system of Hill equation occurs when the excitation frequency $\Omega$ is close to critical values $\left[\omega_{k}(j)+\omega_{k^{\prime}}(j)\right] /(2 n)$ where $\omega_{k}(j)\left(k, k^{\prime}=1,2\right)$ are the eigenfrequencies of Eq. (16) in the nonmodulated case, and $n=1,2, \ldots$.

In our paper we are interested in the case when the modulation frequency $\Omega \gg 2 \sup _{j}\left[\omega_{k}(j)\right]$ and there is no parametric resonance instability. In this case one can average Eqs. (5) with respect to fast oscillations (see Appendix A for details based on a Kapitza approach [32]) and obtain instead of (11) the following equation

$$
\tau \ddot{\psi}_{n}+\dot{\psi}_{n}=A\left(\psi_{n+1}-\psi_{n}\right)-B\left(\psi_{n+2}-2 \psi_{n+1}+\psi_{n}\right),
$$

where the coefficients $A$ and $B$ are given by (see Appendix A for details)

$$
\begin{gathered}
A=\mathcal{A}^{\prime}(\delta) \equiv \frac{\Omega}{2 \pi} \int_{0}^{2 \pi / \Omega} \mathcal{V}^{\prime}(\delta-\eta(t)) d t \\
B=\frac{\tau}{2\left(1+\Omega^{2} \tau^{2}\right)}\left(\mathcal{B}_{1}^{\prime}(\delta)\right)^{2} \\
\equiv \frac{\tau}{2\left(1+\Omega^{2} \tau^{2}\right)}\left[\frac{\Omega}{\pi} \int_{0}^{2 \pi / \Omega} \mathcal{V}^{\prime}(\delta-\eta(t)) \cos (\Omega t) d t\right]^{2},
\end{gathered}
$$

respectively. Note that for $f \rightarrow 0$ the coefficient $A$ is finite while $B \propto f^{2}$.

From Eqs. (17) we obtain

$$
\tau \ddot{\Psi}_{j}(t)+\dot{\Psi}_{j}(t)=\gamma_{j}\left(A-B \gamma_{j}\right) \Psi_{j}(t) .
$$

The solution of Eq. (20) has the form

$$
\Psi_{j}=A_{j}^{+} e^{z_{j}^{+} t}+A_{j}^{-} e^{z_{j}^{-} t},
$$

where

$$
z_{j}^{ \pm}= \pm i \omega_{j}+\lambda_{j}^{ \pm}
$$

are the roots of the equation

$$
\tau z^{2}+z-A \gamma_{j}+B \gamma_{j}^{2}=0 .
$$

Proceeding in the same way as in the nonmodulated case, one may conclude that in the presence of the safety distance time modulation the uniform flow (8) loses its stability when $\operatorname{Re}\left(z_{j}^{+}\right) \geqslant 0$ or equivalently

$$
\begin{aligned}
2 \tau & {\left[A+4 B \sin ^{2}\left(\frac{j \pi}{N}\right)\right]^{2} \cos ^{2}\left(\frac{j \pi}{N}\right) } \\
> & A-2 B \cos \left(\frac{2 j \pi}{N}\right) .
\end{aligned}
$$

In the framework of the optimal velocity model with $\mathcal{V}(u)$ given by (see, e.g., [18])

$$
\mathcal{V}(u)=\tanh (u),
$$

the coefficients $A$ and $B$ become

$$
\begin{aligned}
A_{\mathrm{ov}}= & \frac{1}{8} \operatorname{sech}^{2} \delta\left(8+4 f^{2}\left(2-3 \operatorname{sech}^{2} \delta\right)\right. \\
& \left.+f^{4}\left(2-15 \operatorname{sech}^{2} \delta+15 \operatorname{sech}^{4} \delta\right)\right), \\
B_{\mathrm{ov}}= & \frac{2 f^{2} \tau}{1+\Omega^{2} \tau^{2}} \operatorname{sech}^{4}(\delta) \tanh ^{2}(\delta),
\end{aligned}
$$

and the instability domain for the lowest mode $j=1$ is given by the equation

$$
\begin{aligned}
& 2 \tau\left[A_{\mathrm{ov}}+4 B_{\mathrm{ov}} \sin ^{2}\left(\frac{\pi}{N}\right)\right]^{2} \cos ^{2}\left(\frac{\pi}{N}\right) \\
& >A_{\mathrm{ov}}-2 B_{\mathrm{ov}} \cos \left(\frac{2 \pi}{N}\right) .
\end{aligned}
$$




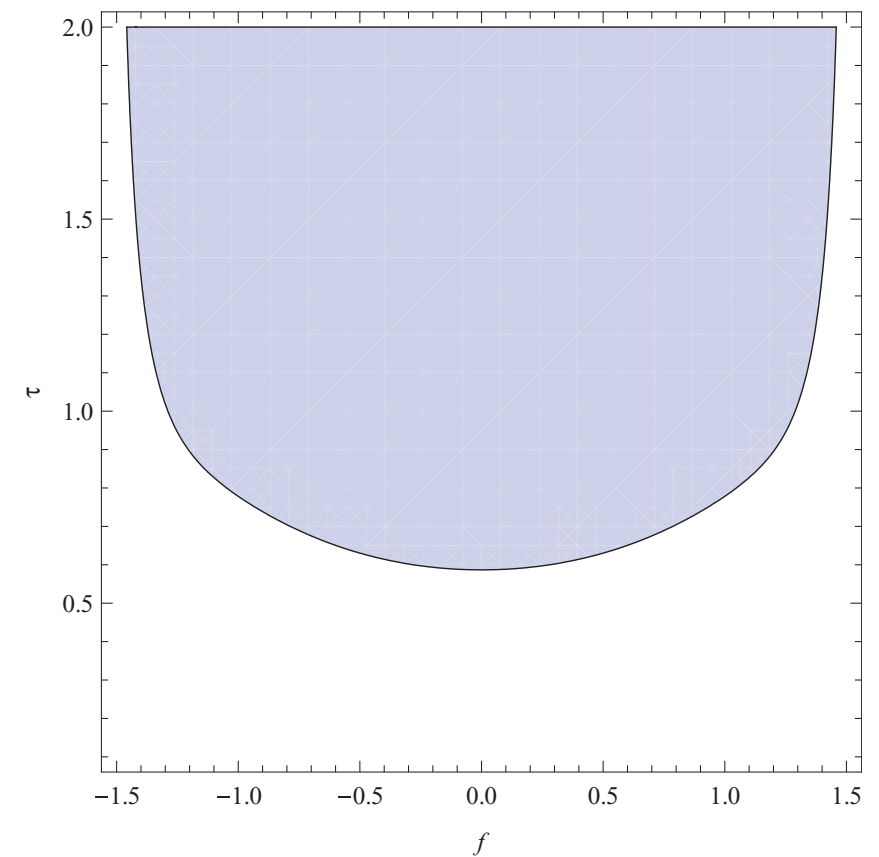

FIG. 1. (Color online) Free flow instability domain (shadow region) for the driven optimal velocity model with fixed car density $\rho=1.7$ in the space of parameters: the amplitude of modulation $f$ and the dimensionless relaxation time $\tau$. Taking into account that the relaxation time $\tau$ is proportional to the speed limit $v: \tau=v T / \ell_{0}$, the diagram shows that the safety distance time modulation makes the free flow stable for larger values of car velocity. Length of the road is $L=30$, driving frequency is $\Omega=5$.

The free flow stability phase diagrams obtained from Eqs. (26) and (27) are presented in Figs. 1 and 2. As it is seen from Fig. 1 the critical value of the dimensionless relaxation time $\tau$ increases when the amplitude of the safety distance $f$ increases. As it follows from Eq. (4) the relaxation time $\tau$ is proportional to the amplitude of the optimal velocity $V$ : $\tau=V T / \ell_{0}$. Thus, the diagram shows that the safety distance time modulation makes the free flow stable for larger values of the car velocity.

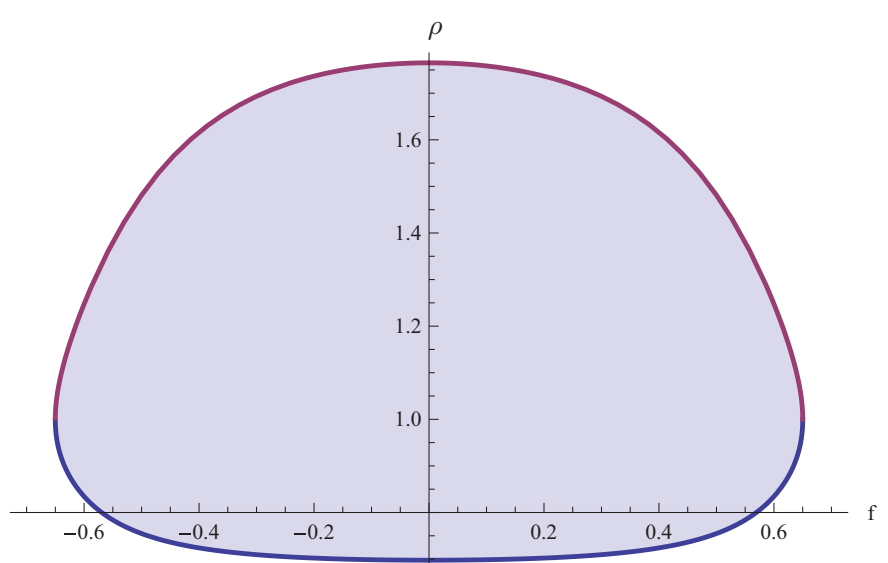

In the space of parameters $(\rho, f)$ the boundary of the free flow instability area is either a convex (Fig. 2, left panel) or concave curve (Fig. 2, right panel) depending on the relaxation time $\tau$ (or, in other words, on the velocity $V$ ). In the limit $L, N \gg 1$ the upper (bottom) boundary is a convex (concave) curve for

$$
\Omega^{2} \tau^{2}(4 \tau-3)+12 \tau-7<0 .
$$

In the case of the opposite inequality the sign of the curvature changes. When the inequality (28) holds the safety time modulation expands the area of free flow stability. This is demonstrated by the left panel of Fig. 2, obtained from Eq. (27) for the reaction time $\tau=0.6$ and the modulation frequency $\Omega=5$. As it is seen from Fig. 2 the density interval in which the uniform flow is unstable shrinks as the absolute value of the modulation amplitude $f$ increases. The right panel of Fig. 2 shows that for the same modulation frequency but with the reaction time $\tau=1.2$ the free flow instability area expands.

To validate the results of our analysis we carried out fullscale numerical simulations of Eqs. (5), (6), (25) and obtained the free flow instability phase diagram (force amplitude $f$, car density $\rho$ ) presented in Fig. 3. The figure shows that the numerical results are in good agreement with the analytical results. It is instructive to present the inequality (28), which separates the regimes of stabilization and destabilization of the free flow in the physical variables. In these variables inequality (28) takes the form

$$
\begin{gathered}
V<V_{c}, \\
V_{c}=\frac{\ell_{0}}{T} \frac{7+3 \omega^{2} T^{2}}{12+4 \omega^{2} T^{2}} .
\end{gathered}
$$

Taking into account that $V$ is the maximal velocity gain, one may conclude that the time modulation enhances stability of the free flow regime for such traffic rules.

If the traffic rules allow a driver to speed up too much, we don't expect that the time modulation of the safety distance will enhance the free flow stability. In the opposite case, the density interval of the free flow stability increases in the presence of the time modulation.

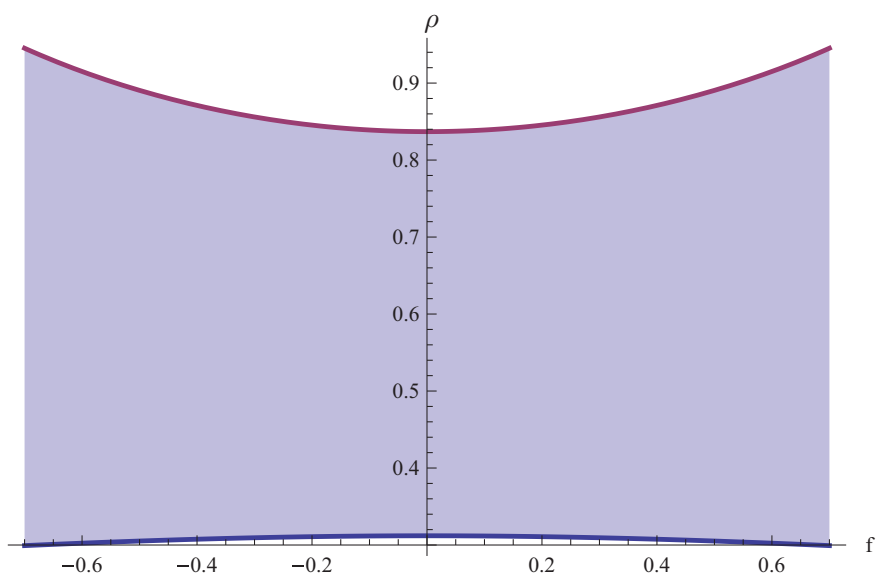

FIG. 2. (Color online) Free flow instability domain (shadow region) for the driven optimal velocity model with fixed reaction time $\tau=0.6$ (left panel) and with $\tau=1.2$ (right panel). Length of the road is $L=30$, driving frequency is $\Omega=5$. 

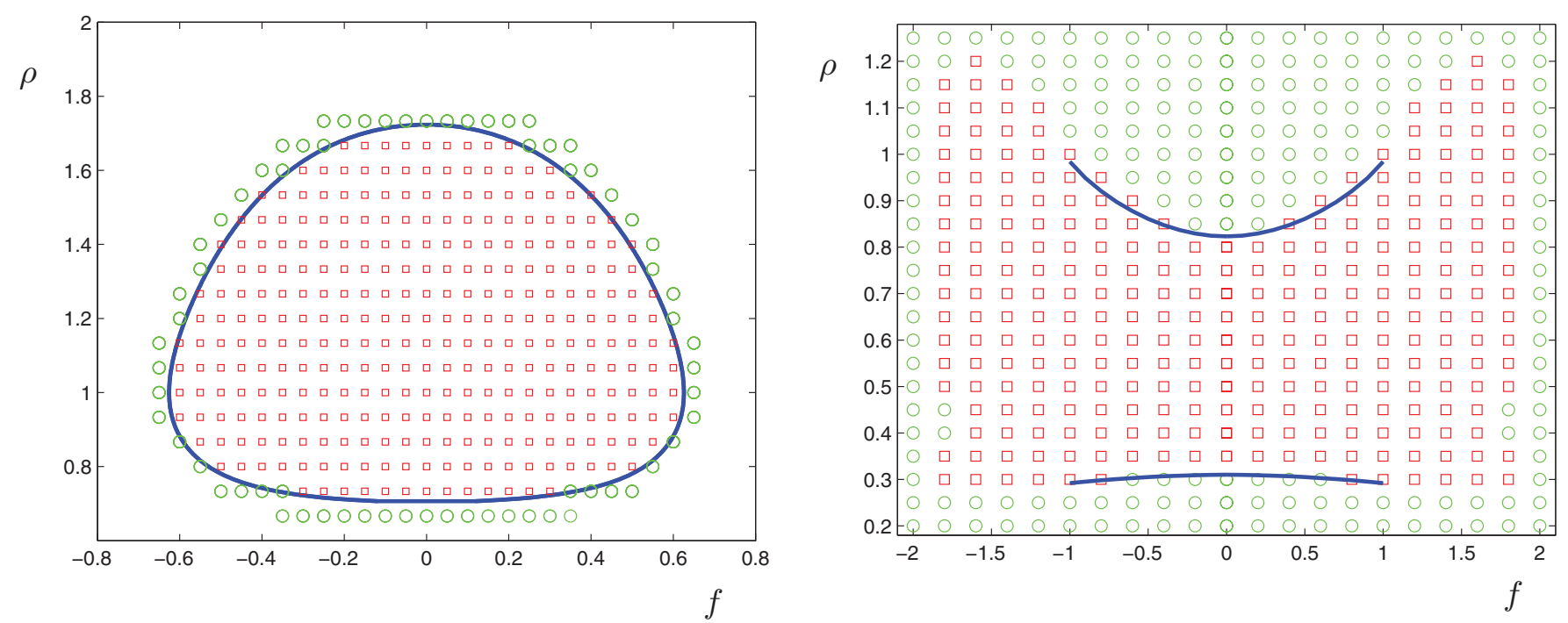

FIG. 3. (Color online) Free flow instability in the $(f, \rho)$ domain obtained from full-scale numerical simulations of the driven optimal velocity model for driving frequency $\Omega=5$ and two values of the reaction time $\tau=0.6$ (left panel) and $\tau=1.2$ (right panel). The region of instability of the free flow is marked by squares and the region of stability is indicated by circles. The analytically obtained boundary of the free flow stability region is given by a solid curve.

\section{FLUX OF THE FLOW}

The aim of this section is to link a microscopic description based on the equations of motion of the optimal velocity model (5) to the corresponding macroscopic one. In order to achieve this we introduce the microscopic flux and the microscopic density given by (see also Ref. [33]).

$$
\hat{J}(x, t)=\sum_{n} \dot{s}_{n}(t) \delta\left(x-s_{n}(t)\right)
$$

and

$$
\hat{\rho}(x, t)=\sum_{n} \delta\left(x-s_{n}(t)\right)
$$

respectively. Here $\delta(\cdot)$ denotes Dirac's $\delta$ function. It can be verified that the functions $\hat{\rho}(x, t)$ and $\hat{J}(x, t)$ satisfy the continuity equation

$$
\partial_{t} \hat{\rho}+\partial_{x} \hat{J}=0 .
$$

The macroscopic flux is linked to the microscopic flux by the expression

$$
J(t)=\frac{1}{L} \int_{0}^{L} \hat{J}(x, t) d x=\rho V(t),
$$

where we have introduced the mean velocity

$$
V(t)=\frac{1}{N} \sum_{n} \dot{s}_{n}(t)
$$

and the mean density of cars, $\rho=N / L=1 / \ell$. In the case of time-modulated traffic it is reasonable to introduce a quantity that would eliminate fast varying variables. This quantity is the mean macroscopic flux $\bar{J}$ can now be defined as a time average of the mean flux

$$
\bar{J}=\langle J(t)\rangle,
$$

where

$$
\langle f(t)\rangle=\frac{1}{\Delta} \int_{t}^{t+\Delta} f\left(t^{\prime}\right) d t^{\prime}, \quad \Delta \rightarrow \infty
$$

denotes a time averaging. Note that this quantity, $\bar{J}$, is widely used in the literature where problems of traffic flux are investigated. flow

From Eqs. (5) and (8) we obtain the flux of the uniform

$$
J_{\mathrm{u}}=\rho\left\langle\mathcal{V}\left(\frac{1}{\rho}-h-\eta(t)\right)\right\rangle+\rho v .
$$

For the safety distance time modulation given by Eq. (6) we get

$$
\begin{aligned}
J_{\mathrm{u}}= & J_{0}+\frac{f^{2}}{8} \rho\left[\left(4+f^{2}\right)-3 f^{2} \operatorname{sech}^{2}\left(h-\frac{1}{\rho}\right)\right] \\
& \times \tanh \left(h-\frac{1}{\rho}\right) \operatorname{sech}^{2}\left(h-\frac{1}{\rho}\right)+\mathcal{O}\left(f^{6}\right),
\end{aligned}
$$

where

$$
J_{0}=\rho\left[v-\tanh \left(h-\frac{1}{\rho}\right)\right]
$$

is the uniform flow flux in the no-modulation case.

Thus by applying the safety distance time modulation, an enhancement of the flux of the uniform flow can be achieved for high-density traffic, namely $\rho h>1$. On the other hand, when the density of cars is small, i.e., for $\rho h<1$, the safety distance time modulation causes the uniform flow flux to decrease.

\section{NUMERICAL INVESTIGATIONS OF TIME-MODULATED TRAFFIC FLOWS}

To investigate how the safety distance time modulation influences the congested regime of traffic flow we performed 


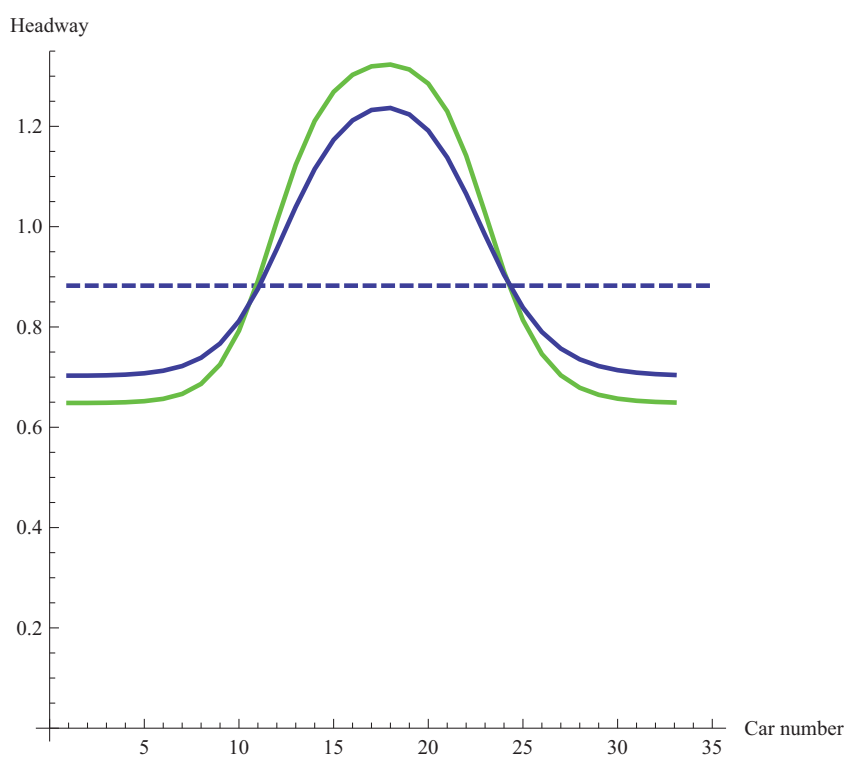

FIG. 4. (Color online) The headway, i.e., distance $x_{n+1}(t)-x_{n}(t)$ between neighboring cars, vs car number $n$. The dashed line corresponds to equidistant uniform car flow, the green (light gray) solid curve to nonmodulated case, solid blue (dark gray) curve to modulation with $f=0.165, \Omega=2$. Parameters: $\tau=0.525, L=30$, $N=34$.

several numerical studies. The equations (5) under the boundary conditions (7) with the optimal velocity function given by the expression (25) and the modulation force $\eta(t)$ in the form (6) were solved numerically using a Runge-Kutta solver. The length of the road was chosen to be $L=30$ and the safety distance $h=1$. A typical headway profile obtained for $\tau>\tau_{c}$ is presented in Fig. 4. The cars in the rarefaction region where $s_{n+1}(t)-s_{n}(t)>\ell$ move faster than in the jam region where $s_{n+1}(t)-s_{n}(t)<\ell$. While the travel direction of the cars is counterclockwise, the wave fronts which separate the rarefaction and jam regions move clockwise (see, e.g., Ref. [30]).

In Fig. 5 we compare the flux dependence on the car density in the nonmodulated case $(f=0)$ to the case where the safety distance is modulated ( $f=0.4, \Omega=2$ ). In the case of highdensity traffic $(\rho>1 / h)$, the flux increases for increasing modulation amplitude and decreases in the opposite case. In other words, the safety distance time modulation facilitates a car propagation in the case when the mean distance between neighboring cars is small and hinders it when the cars in the flow are well separated. For time-modulated safety distance, a pronounced maximum of the flux $J$ of high-density flows $(\rho h>1)$ is observed when changing the modulation strength $f$, as shown in Fig. 6. In contrast, $J(f)$ has a minimum for low-density flows $(\rho h<1)$.

\section{DYNAMICS OF THE TRAFFIC FLOW IN AN INTERACTING MODE APPROACH}

To give some insight into a mechanism of the traffic control we consider a simplified polynomial model in which the

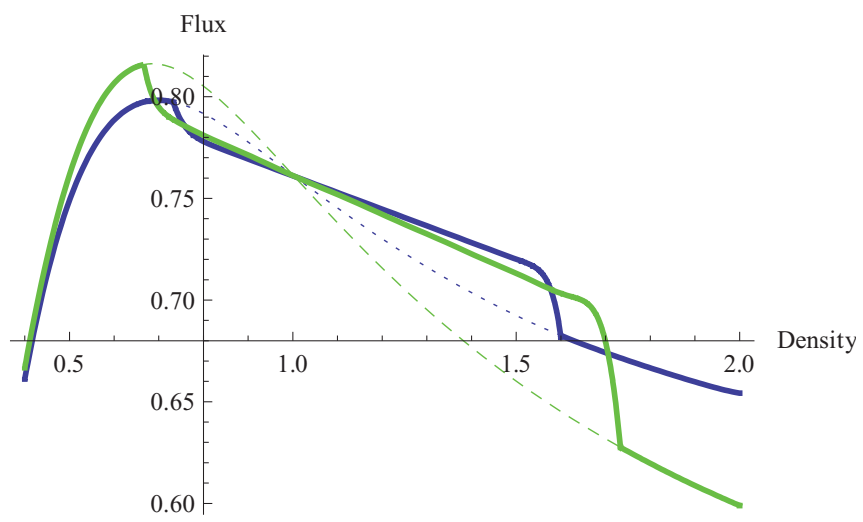

FIG. 5. (Color online) The fundamental diagram for traffic flow: Flow flux $\bar{J}$ vs car density $\rho$ in the modulation case $f=0.4$ [blue (dark gray) curve] and in the nonmodulated case [green (light gray) curve] obtained from full-scale numerical simulations. The solid curves indicate stable solutions, the dashed curves indicate unstable ones. Parameters: $L=30, \tau=0.6$.

optimal velocity function $\mathcal{V}(\delta+q)$ has the form

$$
\mathcal{V}(\delta+q)=\mathcal{V}(\delta)+\frac{1}{2 \tau_{c}}\left(q-\frac{1}{2} \alpha q^{2}-\frac{1}{3} \beta q^{3}\right),
$$

where $\alpha$ and $\beta$ depend on $\delta$. For example, this form can be obtained from the optimal velocity function (25), by assuming that the safety distance modulation is weak: $|\eta(t)| \ll 1$ and the deviation of the headway $s_{n+1}-s_{n}$ from the mean distance $\ell$

$$
u_{n}=s_{n+1}-s_{n}-\ell
$$

is small: $\left|u_{n}\right| \ll 1$. Under these assumptions, by expanding the optimal velocity function (25) into a Taylor polynomial we get

$$
\tau_{c}=\frac{\cosh ^{2}(\delta)}{2}, \quad \alpha=2 \tanh (\delta), \quad \beta=\operatorname{sech}^{2}(\delta)-2 \tanh ^{2}(\delta) .
$$

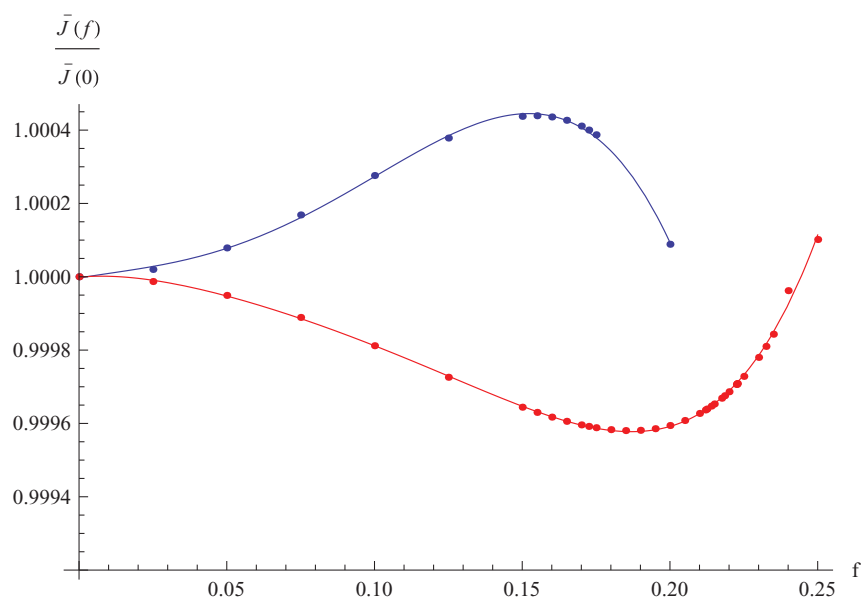

FIG. 6. (Color online) Normalized congested flow flux

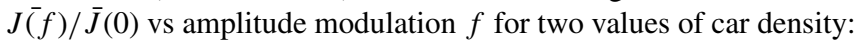
$\rho=1.13$ [blue (upper) curve] and $\rho=0.85$ [red (lower) curve]. Parameters: $L=30, \tau=0.525$. 
Inserting Eqs. (41) into Eq. (5) results in

$$
\begin{gathered}
\tau \ddot{s}_{n}+\dot{s}_{n}=\mathcal{V}(\delta)+v+\frac{1}{2 \tau_{c}}\left(q_{n}-\frac{\alpha}{2} q_{n}^{2}-\frac{\beta}{3} q_{n}^{3}\right), \\
n=1,2, \ldots, N
\end{gathered}
$$

where $q_{n}=s_{n+1}-s_{n}-\ell-\eta(t)$. By subtracting pairwise, we obtain from Eqs. (44) that equations for the functions (42) take the form

$$
\begin{aligned}
2 \tau_{c}\left(\tau \ddot{u}_{n}+\dot{u}_{n}\right)= & \left(u_{n+1}-u_{n}\right)\left(1-\beta \eta^{2}(t)\right) \\
& -\frac{\alpha}{2}\left(u_{n+1}^{2}-u_{n}^{2}\right)-\frac{\beta}{3}\left(u_{n+1}^{3}-u_{n}^{3}\right) \\
& +\left[\alpha\left(u_{n+1}-u_{n}\right)+\beta\left(u_{n+1}^{2}-u_{n}^{2}\right)\right] \eta(t) .
\end{aligned}
$$

It makes sense to introduce quantities that characterize integral features of the flow. These are moments of the car distribution. The second moment

$$
M_{2}=\frac{1}{N} \sum_{n}\left(s_{n+1}(t)-s_{n}(t)-\ell\right)^{2}
$$

gives a mean-square deviation of the headway $s_{n+1}(t)-s_{n}(t)$ (or a variance of headway) from the free flow solution. The quantity

$$
M_{3}=\frac{1}{N} \sum_{n}\left(s_{n+1}(t)-s_{n}(t)-\ell\right)^{3}
$$

is the third moment (skewness) of the car distribution and characterizes its asymmetry. The variance vanishes $\left(M_{2}=0\right)$ for the free (uniform) flow. In the flow with $M_{2} \neq 0$ some of the vehicles are closer to each other than the mean distance $\ell$, others are more separated. In flows with $M_{2} \neq 0$ regions with higher and lower density of cars exist, or in other words there are traffic jams.

As it is seen from Eqs. (44), (46), and (47) the macroscopic flux (34) satisfies the equation

$$
\begin{aligned}
\tau \dot{J}(t)+J(t)= & \rho\left[\mathcal{V}\left(\frac{1}{\rho}-h\right)+v\right] \\
& +\frac{1}{2 \tau_{c} L} \sum_{n}\left(q_{n}-\frac{\alpha}{2} q_{n}^{2}-\frac{\beta}{3} q_{n}^{3}\right) \\
= & J_{\mathrm{u}}(t)-\frac{\rho}{2 \tau_{c}}\left[\left(\frac{\alpha}{2}+\beta \eta(t)\right) M_{2}+\frac{\beta}{3} M_{3}\right],
\end{aligned}
$$

where

$$
J_{\mathrm{u}}(t)=\rho\left[\mathcal{V}\left(\frac{1}{\rho}-h\right)+v-\frac{1}{2 \tau_{c}}\left(\eta-\frac{\alpha}{2} \eta^{2}-\frac{\beta}{3} \eta^{3}\right)\right]
$$

is the macroscopic flux corresponding to the uniform flow. From the definition (36) and Eq. (48) it follows that the mean macroscopic flux $\bar{J}$ can be expressed also in terms of the car distribution moments as

$$
\bar{J}=J_{u}-\frac{\rho}{2 \tau_{c}}\left(\frac{\alpha}{2}\left\langle M_{2}\right\rangle+\frac{\beta}{3}\left\langle M_{3}\right\rangle\right),
$$

where the relation

$$
\left\langle\eta(t) M_{2}\right\rangle \approx 0
$$

is taken into account.

\section{TIME-AVERAGED TRAFFIC DYNAMICS}

As it was mentioned in Sec. II we are interested in the case when the time modulation of the safety distance is fast, i.e., $\Omega \tau \gg 1$. In this case one can apply an averaging with respect to fast oscillations and derive effective equations, which describe the effective dynamical behavior of the system. The corresponding derivation of averaged equations of motion for the general case of the optimal velocity model is presented in Appendix A. By applying this approach to the polynomial model (41), one can obtain [see Eq. (A13) in Appendix A] that the averaged dynamics of the traffic flow with the fast time-modulating safety distance is governed by equations

$$
\begin{aligned}
2 \tau_{c}\left(\tau \ddot{U}_{n}+\dot{U}_{n}\right)= & \left(1-\frac{1}{2} \beta f^{2}\right)\left(U_{n+1}-U_{n}\right) \\
& -\frac{1}{2} \xi f^{2}\left(U_{n+2}-2 U_{n+1}+U_{n}\right) \\
& -\frac{\alpha}{2}\left(U_{n+1}^{2}-U_{n}^{2}\right)-\frac{\beta}{3}\left(U_{n+1}^{3}-U_{n}^{3}\right),
\end{aligned}
$$

where

$$
U_{n}(t)=\left\langle u_{n}(t)\right\rangle
$$

is a headway averaged with respect to fast oscillation, and the notation

$$
\xi=\frac{\alpha^{2} \tau}{2 \tau_{c}\left(1+\Omega^{2} \tau^{2}\right)}
$$

is introduced. Note that for the sake of simplicity in Eq. (51) the last two terms in the right-hand side of Eq. (A13) were neglected. We carried out full-scale numerical simulations of Eqs. (41), (A2) with the safety distance time modulation $\eta(t)=f \cos (\Omega t)$ and numerical simulations of averaged equations (52) for different values of the modulation frequency $\Omega$ and found out that for $\Omega \tau>1$ the results of both types of simulations agree very well (see Appendix A).

Equations (51) show why the time modulation of the safety distance provides an effective control of the traffic flow dynamics. The influence of the time modulation of the safety distance on the averaged traffic dynamics is twofold:

(i) It leads to an effective increasing of the critical value of the reaction time: $\tau_{c} \rightarrow \tau_{c} /\left(1-0.5 \beta f^{2}\right)$.

(ii) It provides a coupling to the next-nearest neighbor.

By expanding the function $U_{n}(t)$ into the Fourier series

$$
\begin{aligned}
& U_{n}(t)=\sum_{j=-\frac{N}{2}+1}^{\frac{N}{2}} Q_{j}(t) \exp \left\{i \frac{2 \pi j n}{N}\right\}, \\
& Q_{j}(t)=\frac{1}{N} \sum_{i=1}^{N} U_{n}(t) \exp \left\{-i \frac{2 \pi j n}{N}\right\},
\end{aligned}
$$


one can present Eqs. (51) in terms of collective modes of the system $Q_{j}(t)$

$$
\begin{aligned}
2 \tau_{c}\left(\tau \ddot{Q}_{j}+\dot{Q}_{j}\right)= & \gamma_{j}\left[\left[\left(1-\frac{\beta}{2} f^{2}\right)-\frac{1}{2} \xi f^{2} \gamma_{j}\right] Q_{j}\right. \\
& -\frac{1}{2} \alpha \sum_{j_{1}, j_{2}} Q_{j_{1}} Q_{j_{2}} \delta_{j, j_{1}+j_{2}} \\
& \left.-\frac{\beta}{3} \sum_{j_{1}, j_{2}, j_{3}} Q_{j_{1}} Q_{j_{2}} Q_{j_{3}} \delta_{j, j_{1}+j_{2}+j_{3}}\right],
\end{aligned}
$$

where $\delta_{j, j^{\prime}}$ is the Kronecker $\delta$. Note that the quantities $U_{n}$ are real and therefore the amplitudes $Q_{j}$ satisfy the conditions $Q_{j}=Q_{-j}^{*}$.

In the linear approximation the solution of Eqs. (55) has the form (21)-(23). Taking into account that for the polynomial model (41)

$$
A=\frac{1}{2 \tau_{c}}\left(1-\frac{\beta}{2} f^{2}\right), \quad B=\frac{\xi f^{2}}{4 \tau_{c}},
$$

we obtain that the modes $j$ satisfying the inequality

$$
\begin{gathered}
\tau \geqslant T_{j}(f), \\
T_{j}(f)=\tau_{c} \sec ^{2}\left(\frac{\pi j}{N}\right) \frac{1-\frac{1}{2} \beta f^{2}-\xi f^{2} \cos \left(\frac{2 \pi j}{N}\right)}{\left[1-\frac{1}{2} \beta f^{2}+2 \xi f^{2} \sin ^{2}\left(\frac{\pi j}{N}\right)\right]^{2}}
\end{gathered}
$$

are linearly unstable.

In our analytical approach we consider the dynamics of the traffic flow near the threshold of the free flow instability:

$$
\tan ^{2}\left(\frac{\pi}{N}\right)<\frac{\tau}{\tau_{c}}-1<\tan ^{2}\left(\frac{2 \pi}{N}\right),
$$

when in the nonmodulated case $(f=0)$ only the mode with $|j|=1$ is linearly unstable while the modes with $|j|>1$ are linearly stable.
Assuming that the number of cars $N$ is large enough such that $\tan \left(\frac{2 \pi}{N}\right) \equiv \epsilon \ll 1$, one can present equations for the linearly unstable mode $(j=1)$ in the form

$$
\begin{aligned}
2 \tau_{c}\left(\tau \ddot{Q}_{1}+\dot{Q}_{1}\right)= & \gamma_{1}\left[\left(1-\frac{\beta}{2} f^{2}-\frac{1}{2} \xi f^{2} \gamma_{1}\right) Q_{1}\right. \\
& \left.-\alpha Q_{1}^{*} Q_{2}-\beta\left(\left|Q_{1}\right|^{2}+2\left|Q_{2}\right|^{2}\right) Q_{1}\right],
\end{aligned}
$$

where the contributions $Q_{j_{1}}^{l} Q_{j_{2}}^{m}(l, m \geqslant 1)$ from the modes with $\left|j_{1,2}\right|>2$ were neglected because their consideration leads to higher-order terms in $\epsilon$.

The linearly stable modes are slaved to the unstable ones and the equation for the most dominant of them reads

$$
\begin{aligned}
2 \tau_{c}\left(\tau \ddot{Q}_{2}+\dot{Q}_{2}\right)= & \gamma_{2}\left[\left(1-\frac{\beta}{2} f^{2}-\frac{1}{2} \xi f^{2} \gamma_{2}\right) Q_{2}\right. \\
& \left.-\alpha Q_{1}^{2}-\beta\left(\left|Q_{2}\right|^{2}+2\left|Q_{1}\right|^{2}\right) Q_{2}\right] .
\end{aligned}
$$

It is seen from Eq. (60) that $Q_{2} \sim Q_{1}^{2} \sim \epsilon^{2}$. Thus taking into account contributions not higher than $\epsilon^{4}$, in terms of the complex amplitudes $Q_{j}(j=1,2)$ the second moment (variance) results in

$$
M_{2}=2\left(\left|Q_{1}\right|^{2}+\left|Q_{2}\right|^{2}\right) .
$$

The third moment (skewness) can be presented as

$$
M_{3}=3\left(Q_{1}^{2} Q_{2}^{*}+\text { c.c. }\right) \text {. }
$$

In polar coordinates

$$
Q_{j}(t)=R_{j}(t) e^{i \Phi_{j}(t)},
$$

the equations for the linearly unstable mode $j=1$ and the dominant linear one $(j=2)$ become

$$
\begin{gathered}
\tau\left(\ddot{R}_{j}-R_{j} \dot{\Phi}_{j}^{2}\right)+\dot{R}_{j}+\frac{1}{2 \tau_{c}}\left\{2 \sin ^{2}\left(\frac{\pi}{N} j\right)\left(C_{j}-\beta R_{j}^{2}-2 \beta R_{3-j}^{2}\right) R_{j}\right. \\
\left.+\frac{\alpha}{j}\left[\cos \left(\frac{2 \pi}{N} j-(-1)^{j}\left(\Phi_{2}-2 \Phi_{1}\right)\right)-\cos \left(\Phi_{2}-2 \Phi_{1}\right)\right] R_{3-j} R_{1}\right\}=0, \\
\tau\left(R_{j} \ddot{\Phi}_{j}+2 \dot{R}_{j} \dot{\Phi}_{j}\right)+R_{j} \dot{\Phi}_{j}-\frac{1}{2 \tau_{c}}\left\{\sin \left(\frac{2 \pi}{N} j\right)\left(D_{j}-\beta R_{j}^{2}-2 \beta R_{3-j}^{2}\right) R_{j}\right. \\
\left.-\frac{\alpha}{j}\left[\sin \left(\frac{2 \pi}{N} j-(-1)^{j}\left(\Phi_{2}-2 \Phi_{1}\right)\right)+(-1)^{j} \sin \left(\Phi_{2}-2 \Phi_{1}\right)\right] R_{3-j} R_{1}\right\}=0,
\end{gathered}
$$

where $j=1,2$ and

$$
\begin{aligned}
& C_{j}=1-\frac{1}{2} \beta f^{2}-\xi f^{2} \cos \left(\frac{2 \pi}{N} j\right), \\
& D_{j}=1-\frac{1}{2} \beta f^{2}+2 \xi f^{2} \sin ^{2}\left(\frac{\pi}{N} j\right) .
\end{aligned}
$$

The stationary states of Eqs. (64) and (65) are given by

$$
R_{j}=\text { const. } \quad \Phi_{j}(t)=j \omega t+\chi_{j}, \quad j=1,2,
$$

where the frequency $\omega$ and the phase shift $\chi_{j}$ are time independent. The stationary solution $R_{j}=0$ corresponds to the free flow regime. In this case $M_{2}=M_{3}=0$ and the mean 

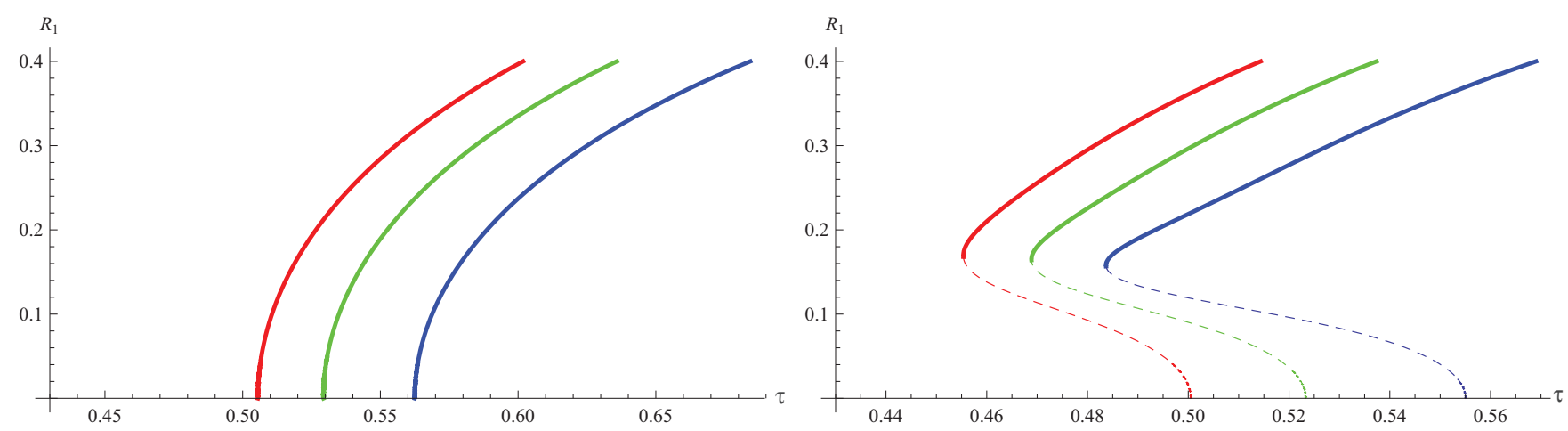

FIG. 7. (Color online) The amplitude of the linearly unstable mode $R_{1}$ vs the time relaxation $\tau$ for two values of car density: $\rho=1$ (left panel) and $\rho=0.9$ (right panel). The dashed curves correspond to unstable branches. From left to right the curves are plotted for safety distance modulation amplitude $f=0, f=0.3$, and $f=0.45$, respectively. Other parameters are $M=30, h=1, \Omega=5$.

flux is given by

$$
J_{\mathrm{u}}=\rho\left[\mathcal{V}\left(\frac{1}{\rho}-h\right)+v+\frac{\alpha}{8 \tau_{c}} f^{2}\right] .
$$

At $\tau=T_{1}(f)$ a bifurcation resulting in a limit cycle occurs with $R_{j}=$ const. $\neq 0$. In terms of the car coordinates $s_{n}(t)$ the periodic orbit (67) corresponds to a traveling wave, which moves with the velocity $c=\frac{N}{2 \pi} \omega$ and is characterized by a finite value of the variance $M_{2}$. In other words, the periodic orbit (67) describes a congested flow. In the general case expressions for the stationary amplitudes $R_{j}$, for the frequency $\omega$ and for the phase difference $2 \chi_{1}-\chi_{2}$ are tedious. In Appendix B we present their derivation. The set of equations (64) and (65) simplifies significantly in the particular case when $\alpha=0$ (the safety distance $h$ coincides with the mean distance $\ell$ ) and the mode with $j=2$ does not couple directly with the linearly unstable mode $j=1$. In this case the problem under consideration is reduced to an equation for the linearly unstable mode. The stationary solution of Eqs. (64) and (65) above the threshold

$$
\tau \geqslant \tau_{c} \frac{\sec ^{2}\left(\frac{\pi}{N}\right)}{1-\frac{1}{2} \beta f^{2}}
$$

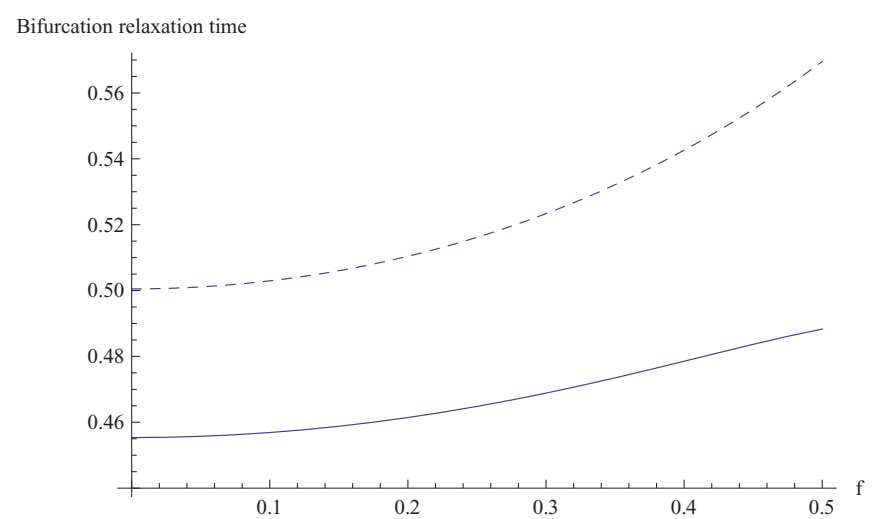

FIG. 8. (Color online) Linear $\tau_{l}$ (dashed line) and nonlinear $\tau_{n l}$ (solid line) bifurcation relaxation time vs modulation amplitude $f$, $(M=30, L=33)$. has the form:

$$
\begin{gathered}
\beta R_{1}^{2}=1-\frac{1}{2} \beta f^{2}-\frac{\tau_{c}}{\tau} \sec ^{2}\left(\frac{\pi}{N}\right), \\
R_{2}=0, \quad \omega=\frac{1}{\tau} \tan \left(\frac{\pi}{N}\right) .
\end{gathered}
$$

By analyzing the stationary solutions of Eqs. (64) and (65) in a general case, one can conclude that the bifurcation to the limit cycle is supercritical for $\rho h=1$ (see Fig. 7, left panel) and subcritical with hysteresis for $\rho h \neq 1$ (see Fig. 7, right panel). There are two characteristic values of the relaxation time $\tau: \tau_{l}$ where a pitchfork bifurcation occurs in $R_{1}$ and $\tau_{n l}$ where a saddle node bifurcation occurs for periodic orbits $\left(\tau_{n l} \leqslant \tau_{l}\right)$. When $\tau \geqslant \tau_{l}$ the free flow loses its stability due to generation of linear Fourier modes [in the considered case it occurs due to generation of the first Fourier harmonics and $\left.\tau_{l} \equiv T_{1}(f)\right]$, while for $\tau \leqslant \tau_{n l}$ the congested flow ceases to exist. Both bifurcation relaxation times increase when the modulation amplitude (see Fig. 8 where the linear bifurcation time $\tau_{l}$ increases by $13 \%$ while the nonlinear one $\tau_{n l}$ increases by $7 \%$ ) as well as the hysteresis interval $\tau_{l}-\tau_{n l}$ increases with the amplitude of the modulation.

The second and third moments of the car distribution as functions of the car density obtained in the interacting mode approach for the nonmodulated case are presented in Fig. 9. In this figure we also compare the same quantities with the numerically computed ones by using the set of equations (5) with the optimal velocity function (25). For small car densities
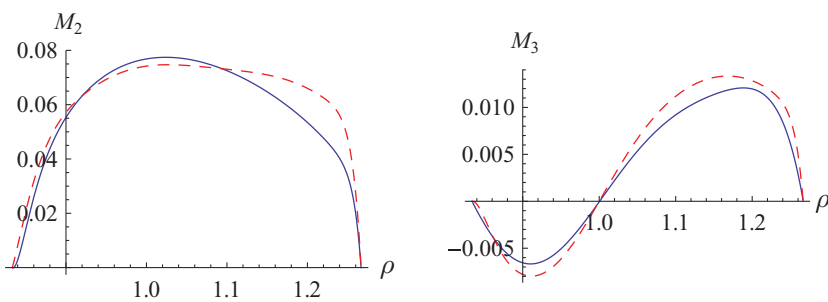

FIG. 9. (Color online) Second and third moments, $M_{2}$ and $M_{3}$, of car distribution vs car density $\rho$ in the nonmodulated case. Solid curves obtained from numerical simulations, dashed curves obtained from Eqs. (61) and (62), i.e., interacting mode approach. Parameters: $L=30, \tau=0.525$. 

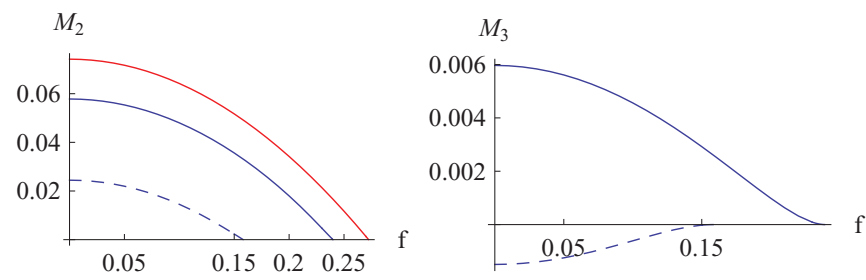

FIG. 10. (Color online) Second and third moments, $M_{2}$ and $M_{3}$, of car distribution $\rho$ vs modulation amplitude $f$ for three values of car density: $\rho=1$ [red solid curve (upper)], $\rho=1.13$ [blue solid curve (lower)], $\rho=0.87$ (dashed curve). Parameters: $L=30, \tau=0.525$, $\Omega=2$.

$(\rho h<1)$ the agreement is quite good while for $\rho h>1$ the results of the interacting mode approach deviate from the full-scale numerical ones. The nonmonotonic behavior of the second and third moments seen in Fig. 9, explains the dependence of the congested flow flux on the density. The third moment $M_{3}$ changes sign at $\rho=1 / h$ for all values of the modulation amplitude $f$. Taking into account that the parameter $\alpha$ in Eq. (50) also changes sign at this density, it explains why the fluxes of the congested flow obtained for different values of the modulation amplitude coincide at the critical density $1 / h$ (see Fig. 5).

In Fig. 10 we analyze the behavior of the second moment $M_{2}$ and the third moment $M_{3}$ for different values of the car density. It is seen that in the presence of the safety distance time modulation the second moment $M_{2}$ and the third moment $M_{3}$ are monotonically decreasing functions of the modulation amplitude. An interplay between the mean-square deviation from the uniform flow, which is expressed by the second moment $M_{2}$ and an asymmetry in the car distribution, which is characterized by the third moment $M_{3}$ provides a nontrivial dependence of the flux of the congestive flow on the strength of safety distance modulation $f$ presented in Fig. 6 .

\section{RELATION TO EXPERIMENTAL DATA}

To clarify if the control mechanism proposed in this paper is viable and efficient we considered two sets of the optimal velocity parameters: the parameters proposed in Ref. [34]

$$
\begin{aligned}
V & =16.8 \mathrm{~m} / \mathrm{s}, \quad v=0.913, \quad T=0.5 \mathrm{~s}, \\
\ell_{0} & =11.63 \mathrm{~m}, \quad H=25 \mathrm{~m}
\end{aligned}
$$

and obtained from real traffic data gathered from Japanese motorways, and the parameters proposed in Ref. [35]

$$
\begin{aligned}
& V=7.91 \mathrm{~m} / \mathrm{s}, \quad v=0.85, \quad T=1.18 \mathrm{~s}, \\
& \ell_{0}=7.7 \mathrm{~m}, \quad H=16 \mathrm{~m},
\end{aligned}
$$

and based on city traffic in Stuttgart.

In accordance with Eq. (4) this means that the dimensionless parameters that control the traffic dynamics are

$$
\begin{gathered}
h=2.15, \quad \tau=0.72, \\
h=2.2, \quad \tau=1.2,
\end{gathered}
$$

respectively.

Inserting Eqs. (71) and (72) into Eq. (30), we see that $V_{c} \in$ $(13.6,17.4) \mathrm{m} / \mathrm{s}$ for the first set and $V_{c} \in(3.8,4.9) \mathrm{m} / \mathrm{s}$ for the

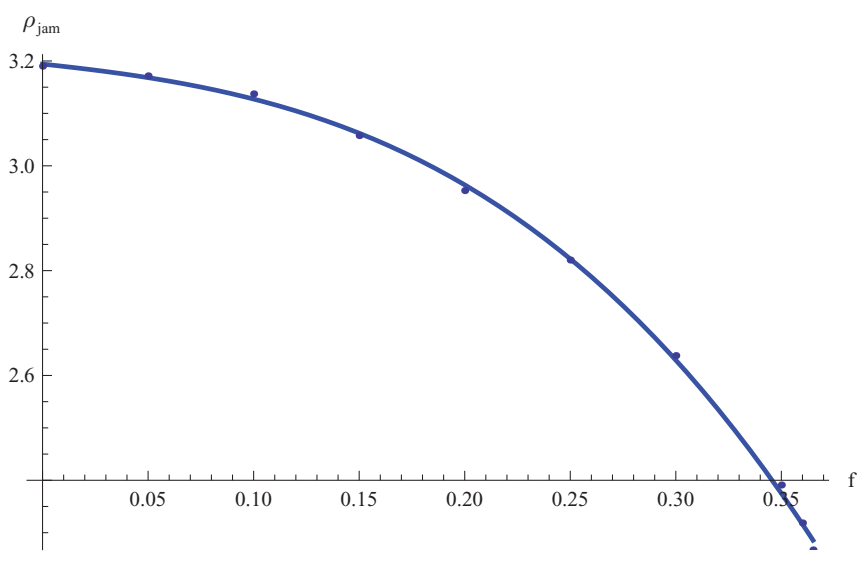

FIG. 11. (Color online) Car density in the jammed region vs amplitude modulation $f$ obtained from full-scale numerical simulations. Parameters: $L=35, N=30, h=2.2, \tau=1.2$.

second one. Invoking Eq. (29) one can conclude while for the set (71) the safety distance time modulation leads to shrinking the area of the uniform flow instability, for the set of parameters given by Eq. (72) one can expect an expansion of the interval of car density $\rho$ where the uniform flow is unstable.

Another interesting characteristic of the traffic, which can be controlled by the safety distance time modulation is the density of cars in a jammed region. We define this quantity as follows:

$$
\rho_{\mathrm{jam}}=\frac{N_{\mathrm{cars}}}{L_{\mathrm{jam}}},
$$

where $N_{\text {cars }}$ is the number of cars in the jammed region and $L_{\text {jam }}$ is the length of the jammed region.

Figure 11 gives an example of the dependence of the density of cars in the jammed region on the safety distance timemodulation amplitude $f$. It is obtained for the set of parameters given by Eq. (72) and shows a well pronounced decrease of density of cars $\rho_{\text {jam }}$ under the action of the time modulation: in the presence of the modulation with the amplitude $f=0.3$ the density in the jammed region decreases by $18 \%$.

\section{CONCLUSIONS}

The main result of the paper is the observation that by applying a time modulation of the safety distance, one can efficiently control the congestive flow formation (traffic jams). It is shown that:

(i) The interval of car density where the uniform (free) flow is unstable changes in the presence of the time modulation compared with the nonmodulated case. It shrinks when the speed-up $V$ is less than some critical value $V_{c}$ and it expands in the opposite case.

(ii) In the interval of densities where the nonuniform (congestive) flow is stable the flux of the time-modulated flow is an increasing function of the amplitude of the modulation for flows whose density is larger than $1 / h$ ( $h$ is the safety distance in the nonmodulated case) and is a decreasing function in the opposite case. In other words, the safety distance time modulation facilitates a car propagation in the case when the mean distance between cars in the congestive traffic is less 
than $h$ and hinders it when the neighboring cars in the flow are well separated.

(iii) The density of cars in the jammed region decreases in the presence of the time modulation compared with the nonmodulated case.

The role of the time modulation is twofold:

(i) It leads to an effective increasing of the critical value of the reaction time.

(ii) It provides an effective coupling to the next-nearest neighbor or, in other words, the time-modulated flow with nearest-neighbor coupling acts equivalently to a flow without time modulation but with coupling also to next-nearest neighbors.

In the framework of interacting modes approach where the traffic jam formation is considered as a limit cycle creation, the safety distance time modulation changes the position of the bifurcation point and modifies the amplitude of the limit cycle (the headway in the congestive flow).

The results of this work are expected to be applicable to a large class of nonlinear systems in which elements are coupled asymmetrically and nonlinearly. The inanimate system composed of camphor boats in an annular water channel [19] offers an interesting possibility for experimental verification of our results.

\section{ACKNOWLEDGMENTS}

The authors thank Toyota Central R\&D Labs, Japan for financial support at the early stage of the work on the project. Yu.G. acknowledges a Guest Professorship funded by grants from Civilingeniør Frederik Christiansens Almennyttige Fond, from IKERBASQUE Foundation (Bilbao, Spain), as well as from the Danish Centre for Applied Mathematics and Mechanics (DCAMM). He also acknowledges partial financial support from a special program of the National Academy of Sciences of Ukraine. Yu.G. is thankful to the Department of Applied Mathematics and Computer Science and the Department of Physics, Technical University of Denmark as well as Department of Applied Mathematics and Statistics, University of the Basque Country for their hospitality.

\section{APPENDIX A}

The aim of this Appendix is to derive from Eqs. (5) with fast oscillating safety distance, equations that describe the averaged effective dynamics of the time-modulated traffic flow. To achieve this, we use a so-called Kapitza's approach [32].

For the headway

$$
u_{n}(t)=s_{n+1}(t)-s_{n}(t)-\ell
$$

we obtain from Eqs. (5) by pairwise subtraction

$$
\tau \ddot{u}_{n}+\dot{u}_{n}=\mathcal{V}\left(u_{n+1}+\delta-\eta(t)\right)-\mathcal{V}\left(u_{n}+\delta-\eta(t)\right),
$$

where $u_{N}=s_{1}-s_{N}-\ell$. In accordance with the boundary conditions (7) we get

$$
u_{n+N}=u_{n} .
$$

The function $\mathcal{V}(u+\delta-\eta(t))$ is a periodic function with period $T=2 \pi / \Omega$. By expanding this function into Fourier series, we can split it in two parts: a time-independent part, $\mathcal{A}$, and a time-dependent one, $\mathcal{B}(t)$

$$
\begin{aligned}
\mathcal{V}(u+\delta-\eta(t)) & =\mathcal{A}(u+\delta)+\mathcal{B}((u+\delta), t), \\
\mathcal{A}(x) & =\frac{\Omega}{2 \pi} \int_{0}^{2 \pi / \Omega} \mathcal{V}(x-\eta(t)) d t, \\
\mathcal{B}(x, t) & =\sum_{n=1}^{\infty} \mathcal{B}_{n}(x) \cos (n \Omega t), \\
\mathcal{B}_{n}(x) & =\frac{\Omega}{\pi} \int_{0}^{2 \pi / \Omega} \mathcal{V}(x-\eta(t)) \cos (n \Omega t) d t .
\end{aligned}
$$

Using this and taking into account only the first harmonics in the expansion for $\mathcal{B}(t)$, Eqs. (A2) can be presented in the following way

$$
\begin{aligned}
\tau \ddot{u}_{n}+\dot{u}_{n}= & \mathcal{A}\left(u_{n+1}+\delta\right)-\mathcal{A}\left(u_{n}+\delta\right)+\left[\mathcal{B}_{1}\left(u_{n+1}+\delta\right)\right. \\
& \left.-\mathcal{B}_{1}\left(u_{n}+\delta\right)\right] \cos (\Omega t) .
\end{aligned}
$$

By presenting the functions $u_{n}(t)$ as a sum

$$
u_{n}(t)=U_{n}(t)+\xi_{n}(t),
$$

where the functions $\xi_{n}(t)$ are small and describe fast oscillations, as we shall see, and $U_{n}(t)$ describes the headway dynamics averaged over the rapid oscillations. Inserting Eq. (A6) into Eqs. (A5) and expanding in powers of $\xi_{n}$, we obtain

$$
\begin{aligned}
\tau \ddot{U}_{n} & +\dot{U}_{n}+\tau \ddot{\xi}_{n}+\dot{\xi}_{n} \\
= & \mathcal{A}\left(U_{n+1}+\delta\right)-\mathcal{A}\left(U_{n}+\delta\right)+\mathcal{A}^{\prime}\left(U_{n+1}+\delta\right) \xi_{n+1} \\
& -\mathcal{A}^{\prime}\left(U_{n}+\delta\right) \xi_{n}+\left[\mathcal{B}_{1}\left(U_{n+1}+\delta\right)\right. \\
& \left.-\mathcal{B}_{1}\left(U_{n}+\delta\right)\right] \cos (\Omega t)+\left[\mathcal{B}_{1}^{\prime}\left(U_{n+1}+\delta\right) \xi_{n+1}\right. \\
& \left.-\mathcal{B}_{1}^{\prime}\left(U_{n}+\delta\right) \xi_{n}\right] \cos (\Omega t),
\end{aligned}
$$

where $A^{\prime}(u) \equiv d A / d u$. By choosing $\xi_{n}$ such that

$$
\tau \ddot{\xi}_{n}+\dot{\xi}_{n}=\left[\mathcal{B}_{1}\left(U_{n+1}+\delta\right)-\mathcal{B}_{1}\left(U_{n}+\delta\right)\right] \cos (\Omega t),
$$

we obtain approximately that the fast oscillating part of the headway is

$$
\begin{aligned}
\xi_{n}(t)= & {\left[\mathcal{B}_{1}\left(U_{n+1}+\delta\right)-\mathcal{B}_{1}\left(U_{n}+\delta\right)\right] } \\
& \times \frac{\sin (\Omega t)-\Omega \tau \cos (\Omega t)}{\Omega\left(1+\Omega^{2} \tau^{2}\right)} .
\end{aligned}
$$

Next, we substitute Eq. (A9) in Eq. (A7) and note that the product $\xi_{n} \cos (\Omega t)$ contains both, time-independent and oscillating terms with frequency $2 \Omega$. The averaging with respect to this fast oscillation with $2 \Omega$, results in the effective dynamics of the traffic flow with time-modulated safety distance, which is described by the equations

$$
\begin{aligned}
\tau \ddot{U}_{n}+\dot{U}_{n}= & \mathcal{A}\left(U_{n+1}+\delta\right)-\mathcal{A}\left(U_{n}+\delta\right)-\frac{\tau}{2\left(1+\Omega^{2} \tau^{2}\right)} \\
& \times\left\{\mathcal { B } _ { 1 } ^ { \prime } ( U _ { n + 1 } + \delta ) \left[\mathcal{B}_{1}\left(U_{n+2}+\delta\right)\right.\right. \\
& \left.-\mathcal{B}_{1}\left(U_{n+1}+\delta\right)\right]-\mathcal{B}_{1}^{\prime}\left(U_{n}+\delta\right)\left[\mathcal{B}_{1}\left(U_{n+1}+\delta\right)\right. \\
& \left.\left.-\mathcal{B}_{1}\left(U_{n}+\delta\right)\right]\right\} .
\end{aligned}
$$

Expanding the left-hand side of Eq. (A10) in powers of $U_{n}$ as far as the first-order terms, we get

$$
\begin{aligned}
\tau \ddot{U}_{n}+\dot{U}_{n}= & \mathcal{A}^{\prime}(\delta)\left(U_{n+1}-U_{n}\right)-\frac{\tau}{2\left(1+\Omega^{2} \tau^{2}\right)}\left(\mathcal{B}^{\prime}(\delta)\right)^{2} \\
& \times\left(U_{n+2}-2 U_{n+1}+U_{n}\right) .
\end{aligned}
$$




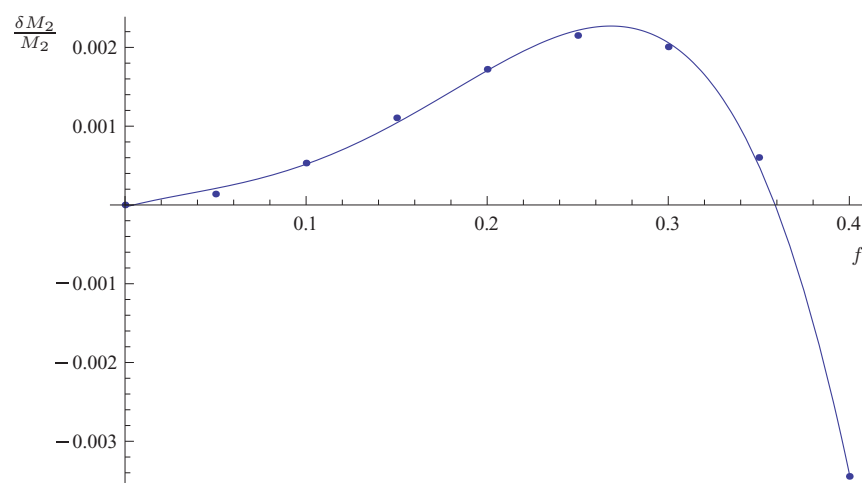

FIG. 12. (Color online) Normalized difference $\frac{\delta M_{2}}{M_{2}}=\frac{M_{2}-\bar{M}_{2}}{M_{2}}$ between second moment $M_{2}$ obtained from full scale numerical simulations of Eqs. (A2), with time modulated safety distance using $\eta(t)=f \cos (2 t)$, and second moment $\bar{M}_{2}$ obtained by the averaging procedure, $v s$ modulation amplitude $f$. Parameters: $L=30, \tau=0.6$, $N=34$. Curve interpolates between 9 computed values.

In the polynomial approach (41) when

$$
\begin{aligned}
\mathcal{A}\left(U_{n}+\delta\right) & =\frac{1}{2 \tau_{c}}\left[\left(1-\frac{1}{2} \beta f^{2}\right) U_{n}-\frac{\alpha}{2} U_{n}^{2}-\frac{\beta}{3} U_{n}^{3}\right], \\
\mathcal{B}_{1}\left(U_{n}+\delta\right) & =\left(\alpha U_{n}+\beta U_{n}^{2}\right) f,
\end{aligned}
$$

the set of equations (A10) takes the form

$$
\begin{aligned}
2 \tau_{c}( & \left.\tau \ddot{U}_{n}+\dot{U}_{n}\right) \\
= & \left(1-\frac{1}{2} \beta f^{2}\right)\left(U_{n+1}-U_{n}\right)-\frac{\alpha}{2}\left(U_{n+1}^{2}-U_{n}^{2}\right) \\
& -\frac{\beta}{3}\left(U_{n+1}^{3}-U_{n}^{3}\right)-\frac{\tau}{4 \tau_{c}\left(1+\Omega^{2} \tau^{2}\right)} f^{2} \\
& \times\left\{\alpha^{2}\left(U_{n+2}-2 U_{n+1}+U_{n}\right)\right. \\
& +\alpha \beta\left[U_{n+2}^{2}-2 U_{n+1}^{2}+U_{n}^{2}+2 U_{n+1}\left(U_{n+2}-U_{n+1}\right)\right. \\
& \left.-2 U_{n}\left(U_{n+1}-U_{n}\right)\right]+\beta^{2}\left[U_{n+1}\left(U_{n+2}^{2}-U_{n+1}^{2}\right)\right. \\
& \left.\left.-U_{n}\left(U_{n+1}^{2}-U_{n}^{2}\right)\right]\right\} .
\end{aligned}
$$

We verified that the results based on averaged equations agree with the full-scale numerical simulations. Figure 12 gives the normalized difference

$$
\frac{\delta M_{2}}{M_{2}}=\frac{M_{2}-\bar{M}_{2}}{M_{2}}
$$

between the second moment $M_{2}$ obtained from the full-scale numerical simulations of Eqs. (A2) and (41) with the safety distance time modulation $\eta(t)=f \cos 2 t$ and the second moment $\bar{M}_{2}$ obtained from Eqs. (A13). For $\Omega=2$ the difference is less than $0.2 \%$. For a modulation frequency $\Omega=1$ the difference is about $1 \%$.

\section{APPENDIX B}

The aim of this Appendix is to study the stationary state of the system of equations (64) and (65). The stationary solution is given by Eq. (67). Inserting Eq. (67) into Eqs. (64) and (65) with $j=1$, we get

$$
\begin{aligned}
& -\zeta o^{2}+C_{1}-\beta R_{1}^{2}-2 \beta R_{2}^{2}=\alpha R_{2} \frac{\sin (k-\chi)}{\sin k}, \\
& -\zeta o+D_{1}-\beta R_{1}^{2}-2 \beta R_{2}^{2}=\alpha R_{2} \frac{\cos (k-\chi)}{\cos k},
\end{aligned}
$$

where the notations

$$
\begin{aligned}
& \chi=2 \chi_{1}-\chi_{2}, \quad k=\frac{\pi}{N}, \quad \zeta=\frac{\tau_{c}}{\tau} \sec ^{2}\left(\frac{\pi}{N}\right), \\
& o=\frac{1}{\tau \omega} \tan \left(\frac{\pi}{N}\right)
\end{aligned}
$$

are introduced. In the same way, from Eqs. (64) and (65) with $j=2$ we find

$$
\begin{aligned}
& -\zeta o^{2}+\left(C_{2}-2 \beta R_{1}^{2}-\beta R_{2}^{2}\right) \cos ^{2} k \\
& =\alpha \frac{R_{2}^{2}}{4 R_{1}} \frac{\sin (2 k+\chi)}{\tan k}, \\
& -\zeta o+\left(D_{2}-2 \beta R_{1}^{2}-\beta R_{2}^{2}\right) \cos (2 k) \\
& =\alpha \frac{R_{2}^{2}}{2 R_{1}} \cos (2 k+\chi) \tan k .
\end{aligned}
$$

Subtraction of Eqs. (B1) and (B2) yields

$$
\zeta o(o-1)+\xi f^{2}=2 \alpha R_{2} \frac{\sin \chi}{\sin (2 k)} .
$$

Multiplying Eq. (B4) by $\cos (2 k)$ and Eq. (B5) by $\cos ^{2} k$ and subtracting them, we get

$$
\begin{aligned}
& \zeta o\left(\cos ^{2} k-o \cos (2 k)\right)-\xi f^{2} \cos ^{2} k \cos (2 k) \\
& =\alpha \frac{R_{1}^{2}}{4 R_{2}} \frac{\sin \chi}{\tan k} .
\end{aligned}
$$

From Eqs. (B6) and (B7) we obtain that

$$
\begin{aligned}
R_{2}^{2}= & \frac{1}{4} R_{1}^{2} \frac{\zeta o(o-1)+\xi f^{2}}{\zeta o\left(\cos ^{2} k-o \cos (2 k)\right)-\xi f^{2} \cos ^{2} k \cos (2 k)} \\
& \times \cos ^{2} k .
\end{aligned}
$$

By dividing Eq. (B1) by Eq. (B2) and Eq. (B4) by Eq. (B5), we get

$$
\begin{aligned}
\frac{-\zeta o^{2}+C_{1}-\beta R_{1}^{2}-2 \beta R_{2}^{2}}{-\zeta o+D_{2}-\beta R_{1}^{2}-2 \beta R_{2}^{2}} \tan k & =\tan (k-\chi), \\
2 \frac{-\zeta o^{2}+\left(C_{2}-2 \beta R_{1}^{2}-\beta R_{2}^{2}\right) \cos ^{2} k}{-\zeta o+\left(D_{2}-2 \beta R_{1}^{2}-\beta R_{2}^{2}\right) \cos (2 k)} \tan k & =\tan (2 k+\chi) .
\end{aligned}
$$

By eliminating $\chi$ from Eqs. (B9) and invoking Eq. (B8), the amplitude of the first mode can be expressed as a fraction

$$
\beta R_{1}^{2}=\frac{W_{1}}{W_{2}},
$$

where

$$
\begin{aligned}
W_{1}= & 2 \zeta^{2}(o-1) o[3+(2 o-1)(o \cos (4 k)-(3+o) \\
& \times \cos (2 k))]-\xi f^{2}\left[\left(-4 o^{2}+6 o-6\right) \cos (2 k)\right. \\
& \left.+\left(o^{2}+o-2\right) \cos (4 k)+o(3 o+5)-4\right] \\
& +2 \xi^{2} f^{4}(1+2 \cos (2 k)) \sin ^{2}(2 k),
\end{aligned}
$$




$$
\begin{aligned}
W_{2}= & 2 \cos (2 k)\left(\zeta o(5 o-3)+3 \xi f^{2}+\xi f^{2} \cos (4 k)\right. \\
& \left.+\zeta(o-3) o+2 \xi f^{2}\right) .
\end{aligned}
$$

Thus, Eqs. (B8) and (B10)-(B12) express the amplitudes $R_{1}$ and $R_{2}$ in terms of the frequency parameter $o$. An equation for the frequency parameter $o$ is obtained by eliminating $\sin (k-\chi)$ and $\cos (k-\chi)$ from Eqs. (B1) and (B2):

$$
\begin{gathered}
\left(-\zeta o^{2}+C_{1}-\beta R_{1}^{2}-2 \beta R_{2}^{2}\right)^{2} \sin ^{2} k+(-\zeta o \\
\left.+D_{1}-\beta R_{1}^{2}-2 \beta R_{2}^{2}\right)^{2} \cos ^{2} k=\alpha^{2} R_{2}^{2},
\end{gathered}
$$

taking into account Eqs. (B8) and (B10)-(B12).
[1] Traffic and Granular Flow, edited by D. E. Wolf, M. Schreckenberg, and A. Bachem (World Scientific, Singapore, 1996).

[2] D. Helbing, Rev. Mod. Phys. 73, 1067 (2001).

[3] T. Nagatani, Rep. Prog. Phys. 65, 1331 (2002).

[4] B. S. Kerner, The Physics of Traffic: Empirical Freeway Pattern Features, Engineering Applications, and Theory (Springer, Heidelberg, 2004).

[5] B. S. Kerner, Introduction to Modern Traffic Flow Theory and Control. The Long Road to Three-Phase Traffic Theory (Springer, Berlin, 2009).

[6] A. Schadschneider, D. Chowdhury, and K. Nishinari, Stochastic Transport in Complex Systems (Elsevier, The Netherlands, 2010).

[7] S. Maerivoet and B. De Moor, Phys. Rep. 419, 1 (2005).

[8] D. Chowdhury, L. Santen, and A. Schadschneider, Phys. Rep. 329, 199 (2000).

[9] A. Takamatsu, R. Tanaka, H. Yamada, T. Nakagaki, T. Fujii, and I. Endo, Phys. Rev. Lett. 87, 078102 (2001).

[10] A. Takamatsu, R. Tanaka, and T. Fujii, Phys. Rev. Lett. 92, 228102 (2004).

[11] A. H. Cohen, P. J. Holmes, and R. H. Rand, J. Math. Biol. 13, 345 (1982).

[12] C. M. A. Pinto and M. Golubitsky, J. Math. Biol. 53, 474 (2006).

[13] V. In, A. Kho, J. D. Neff, A. Palacios, P. Longhini, and B. K. Meadows, Phys. Rev. Lett. 91, 244101 (2003).

[14] K. Nishinari, K. Sugawara, T. Kazama, A. Schadschneider, and D. Chowdhury, Physica A 372, 132 (2006).

[15] K. Nagel and M. Schreckenberg, J. Phys. I 2, 2221 (1992).

[16] M. Treiber, A. Hennecke, and D. Helbing, Phys. Rev. E 62, 1805 (2000).

[17] A. Kesting and M. Treiber, Comput. Aided Civ. Infrastruct. Eng. 23, 125 (2008).
[18] M. Bando, K. Hasebe, A. Nakayama, A. Shibata, and Y. Sugiyama, Phys. Rev. E 51, 1035 (1995).

[19] N. J. Suematsu, S. Nakata, A. Awazu, and H. Nishimori, Phys. Rev. E 81, 056210 (2010).

[20] D. Tanaka, Phys. Rev. Lett. 99, 134103 (2007).

[21] A. Jelic, C. Appert-Rolland, S. Lemercier, and J. Pettre, Phys. Rev. E 85, 036111 (2012).

[22] C. Y. Liang and H. Peng, JSME Int. J. Ser. C 43, 671 (2000).

[23] P. Y. Li and A. Shrivastava, Trasp. Res., Part C: Emerg. Technol. 10, 275 (2002).

[24] S. Kikuchi, N. Uno, and M. Tanaka, J. Trans. Eng. 129, 146 (2003).

[25] A. Bose and P. Ioannou, Trasp. Res. Part C 11, 439 (2003).

[26] A. Kesting, M. Treiber, M. Schönhof, D. Helbing, Transp. Res., Part C: Emerg. Technol. 16, 668 (2008).

[27] K. Konishi, H. Kokame, and K. Hirata, Phys. Rev. E 60, 4000 (1999); Eur. Phys. J. B 15, 715 (2000).

[28] L. C. Davis, Phys. Rev. E 69, 066110 (2004).

[29] http://www.toyota-global.com/innovation/safety_technology/ safety_technology/technology_file/active/radar.html

[30] Yu. B. Gaididei, R. Berkemer, J. G. Caputo, P. L. Christiansen, A. Kawamoto, T. Shiga, M. P. Sørensen, and J. Starke, New J. Phys. 11, 073012 (2009).

[31] A. P. Seyranian and A. A. Mailybaev, Multiparameter Stability Theory with Mechanical Applications (World Scientific, Singapore, 2004).

[32] L. Landau and E. Lifshitz, Mechanics (Pergamon Press, Oxford, 1960).

[33] H. K. Lee, H.-W. Lee, and D. Kim, Phys. Rev. E 64, 056126 (2001).

[34] M. Bando, K. Hasebe, K. Nakanishi, and A. Nakayama, Phys. Rev. E 58, 5429 (1998).

[35] D. Helbing and B. Tilch, Phys. Rev. E 58, 133 (1998). 